\title{
An Innovative Vaccinator Training Program
}

\author{
Gary W. Selnow, $\mathrm{PhD}^{1}$; Charlotte A. Ferretti EdD, RN, FAAN ${ }^{2}$; Maryam Othman, MD, \\ $\mathrm{MPH}^{3}$; Gray Maganga, BEd, $\mathrm{MScIH}^{4}$; William D. Crano, $\mathrm{PhD}^{5}$
}

${ }^{1}$ Claremont Graduate University, Claremont, CA

${ }^{2}$ San Francisco State University, San Francisco, CA

${ }^{3}$ Western University of Health Sciences-COMP, Pomona, CA

${ }^{4}$ Heidelberg University, Heidelberg, Germany

${ }^{5}$ Claremont Graduate University, Claremont, CA

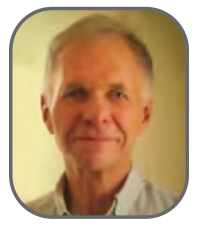

Gary W. Selnow, PhD

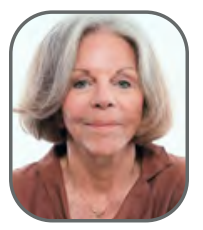

Charlotte A. Ferretti EdD, RN, FAAN

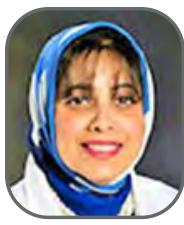

Maryam Othman, MD, MPH

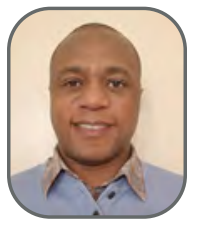

Gray Maganga, BEd, MScIH

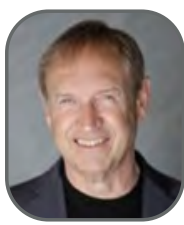

William D. Crano, PhD

\section{CORRESPONDENCE:}

Gary W. Selnow, PhD gary@wiredinternational.org

DOI: $10.38206 / 140106$

KEYWORDS:

COVID-19, Vaccination, Vaccinator Training Program, WiRED

\section{ABSTRACT}

Vaccinations offer the only real resolution to the pandemic. Masks and distance help keep the virus at bay, but they are not long-term solutions. Effective vaccines are now reaching millions in high-income countries, and, in time, they will reach underserved regions. The missing link in the vaccination chain will be trained people to administer them in local communities. Without trained workers, vaccination programs grind to a halt. Large organizations and governments can provide the vaccines, but where will lowresource countries find enough trained people to vaccinate the population?

The objective of the program described here is to train people at the local level to move vaccines safely from central drop-off points to communities, to prepare the communities for vaccinations, to properly screen patients, to administer vaccinations, to monitor patients after the shot for adverse effects following injection (AEFI) and to educate the community on the need to get vaccinated and on vaccine safety. This program trains grassroots vaccinators to perform these tasks and offers refresher training to retired medical professionals called back into service to help with vaccinations.

Each vaccinator will receive 20 hours of WiRED's curriculum based on the World Health Organization's (WHO) vaccination protocols. Further, students will receive an additional 20 hours of instruction on basic health, including human anatomy and physiology, principles of infectious disease control, and coronavirus infections. These frontline workers also will receive an additional five hours of clinical vaccination practice.

\section{BACKGROUND}

By the fourth quarter of 2020, when the COVID-19 case numbers and death counts were on the rise in many countries, more than a dozen vaccines were in the pipeline at various stages of production and distribution. Another 100 vaccines were somewhere within the three testing phases; manufacturers adopted one of four vaccine strategies (whole virus, protein subunit, viral vector, and nucleic acid [RNA and DNA]). ${ }^{1}$ The dominant vaccines were the relatively new messenger RNA (mRNA) vaccines and the more familiar viral vector vaccines. It was a race of production speed and efficacy and the capacity of manufacturers to proceed through early test phases for safety, early effects, and dosage, then eventually to reach Phase 3 clinical trials on large numbers of subjects to test for effectiveness against the virus. 
In mid-December, two vaccines (Pfizer and Moderna), both mRNA, completed Phase 3 trials and cleared rigorous U.S. Government's emergency use authorization. A third vaccine, Janssen's Johnson \& Johnson (J\&J), a viral vector vaccine, followed two months later. The first two vaccines reported remarkable efficacy in the range of $93 \%-95 \%{ }^{2}$ and set public expectations for COVID-19 vaccines. While highly effective by conventional vaccine standards, the third vaccine was reported to be between $67 \%$ and $85 \%$ effective, depending on the measured severity of illness. While the effectiveness of the Janssen viral vector vaccine was slightly lower than the mRNA varieties, it offered two considerable advantages: its cold-chain requirements were more relaxed so that it could be transported more easily, and it required only a single shot, whereas the mRNA vaccines required two shots, several weeks apart.

Despite its virtues, the Janssen vaccine ran into problems early in its release when in mid-April the FDA and the CDC paused its use after reports of a handful of cases of a rare and severe type of blood clot among recipients. The pause allowed scientists at the agencies to review data on the risk of cerebral venous sinus thrombosis (CVST) seen in combination with thrombocytopenia (low blood platelet levels). Following an extensive examination of the data and an analysis that showed the risk was very low, the FDA and CDC recommended a lifting of the pause and a resumption of Janssen vaccinations. With a concurrence of the Advisory Committee on Immunization Practices (ACIP), the vaccinations restarted in late April while the watchdog agencies committed to monitor any new information about adverse effects. ${ }^{3,4,5}$

Remarkably, within less than a year from the arrival of the coronavirus on the global stage, people in the U.S. and several other countries were lining up for vaccinations. Accelerated production and an aggressive U.S. government rollout of the vaccines saw at least three million shots per day in the early spring of 2021.6 The U.S. Secretary of State, Anthony Blinken, predicted that by the end of April 2021, more than 200 million Americans would complete a course of one of the three available vaccines. ${ }^{7}$ The United Kingdom vaccination rates for total vaccinations and percentage of the population vaccinated far surpassed other European countries. ${ }^{8,9}$ Several other early adopters, including Serbia,
TABLE 1.

Vaccines approved by at least one country -April 10,202111

\begin{tabular}{|l|l|l|}
\hline NAME & TYPE & COUNTRIES \\
\hline Anui Zhifel Longcom RBD-Dimer & Protein Subunit & 2 \\
\hline Bharat Ciotech Covaxin & Inactivated & 5 \\
\hline CanSino Ad5-nCoV & Non-replicating v. vector & 4 \\
\hline FBRI EpiVacCorona & Protein Subunit & 2 \\
\hline Gamaleya SputnikV & Non-replicating v. vector & 55 \\
\hline Janssen (J\&J)Ad26.COV2.S & Non-replicating v. vector & 35 \\
\hline Moderna mRNA-1273 & mRNA & 41 \\
\hline Oxford/AstraZeneca AZD1222 & Non-replicating v. vector & 81 \\
\hline Pfizer/BioNTech BNT162b2 & mRNA & 79 \\
\hline Serum Institute of India Covishield & Non-replicating v. vector & 31 \\
\hline Sinopharm (Beijing) BBIBP-CorV & Inactivated & 27 \\
\hline Sinopharm (Wuhan) Inactivated (Vero Cells) & Inactivated & 2 \\
\hline Sinovac CoronaVac & Inactivated & 19 \\
\hline & & \\
\hline
\end{tabular}

Turkey, and Hungary, also quickly distributed vaccines with a plan stratified on age and critical occupations. The U.K. campaign used the two-jab Pfizer and Oxford AstraZeneca vaccines. Other European countries have used a collection of vaccines, including Pfizer, Sputnik $V$, and Sinopharm. ${ }^{10}$

As of spring 2021, there are 100 vaccine candidates in various trial phases and 13 vaccines approved by at least one country. Table 1 lists the approved vaccines, the type and number of countries approving them.

\section{NOT A LOT OF SHARING WITH UNDERSERVED COUNTRIES}

In early February 2021, WHO reported that 90 percent of vaccinations had been administered in high-income countries, with the rest of the world sharing the remaining 10 percent. ${ }^{12}$ The rollout of vaccines has been and likely will continue to be inequitable. The wealthy countries allocate most of the vaccines to themselves first with no clear, early plan for how they will share the surplus with low-resource countries. While individual countries have been inward-looking, a significant transnational effort headed by the Vaccines Global Access (COVAX) and coled by the Coalition for Epidemic Preparedness Innovations (CEPI), Gavi and WHO are working with their delivery partner, UNICEF, to distribute vaccines to Africa and Asia and other 
regions lower down on the distribution list. COVAX is joined by the Pan American Health Organization (PAHO) for procuring vaccines in the Americas. ${ }^{13}$

At the time of this writing, it is unknown when vaccines will reach the needy countries in quantities sufficient to impact the COVID-19 case and death counts. Predictions about distribution depend on research outcomes of the pending vaccines and country acceptance of the vaccines available. Additional vaccine approvals will increase the flow and availability and thus the probability that low-income countries will be able to vaccinate their populations. While the availability of effective and safe vaccines is a critical start of the process, funding and commitment to distribute these vaccines must be in place. This obvious point remains unresolved as supporters scramble to secure international funding and make logistical support provisions, particularly in traditionally inaccessible regions.

Richard Hatchett, CEO of CEPI, estimates that by the end of 2021, more than two billion doses will be available globally, 1.3 billion of which will be allocated to 92 low- and middle-income countries. ${ }^{14}$ These estimates, however, come with considerable cautions because much is unknown about how many vaccines will be effective and approved and how quickly manufacturers can ramp up production. Also, as noted, much depends on funding and resource availability to distribute the vaccines to major urban areas and the countryside. It is essential to inoculate populations if global herd immunity is to be reached. As some observers have noted about COVID-19, "no one is safe until everyone is safe," reflecting on the fact that as long as populations harbor this rapidly mutating virus, there remains a significant chance that variants with the capacity to defeat current vaccines could arise and threaten rich and poor countries alike. ${ }^{15}$

\section{WHEN THE VACCINES ARRIVE}

There remains a good measure of uncertainty about when the vaccines will arrive on the shores of low-income countries and if there will be sufficient quantities of vaccine to accommodate entire populations. Those are the immediate questions at the time of this writing. However, there is one near-certainty that looms in many regions, and that has to do with the local capacity to distribute the vaccines once they arrive. Given the scarcity of doctors and nurses, there is inadequate capacity in many countries to move the vaccines from urban hubs to the outreaches and into the arms of billions of people.

\section{SHORTFALLS OF PHYSICIANS AND NURSES}

Reliable data sources agree that the number of physicians and nurses in low- and middle-income countries is meager under the best of conditions and completely inadequate to accommodate the medical demands imposed by the pandemic. ${ }^{16}$

Routine surveys conducted over the years by $\mathrm{WHO}$, the World Bank, PAHO, and others demonstrate the severity of the problem. For instance, whereas high-income countries have an average of 31 physicians per 10,000 people, lowincome countries have only three per 10,000, and lowermiddle-income countries have eight physicians per 10,000. ${ }^{17}$ Similarly, where high-income countries have 110 practicing nurses per 10,000 people, low-income countries have nine nurses per 10,000, and lower-middle-income countries have 18 nurses per 10,000. ${ }^{17}$ The disparity in numbers of physicians and nurses between rich and poor countries is stunning, and the measures are reasonably consistent across regions with income as the determining factor.

For years, WHO has been well aware of these inequities and has explored strategies to boost healthcare workforces in underserved regions. One approach has been to increase the number and expand the role of community health workers (CHWs), but that has not met great success. Still, CHWs represent the only option to expand the vaccination workforce in most countries. With COVID-19 looming and the need for vaccinators becoming clearer as vaccines move through development and clinical trials toward delivery, WHO anticipates a vaccination bottleneck. Accordingly, it has created a vaccinator training program to teach the paraprofessional CHW corps the skills necessary to assist physicians and nurses in administering COVID-19 vaccines. ${ }^{18}$

Appropriately training $\mathrm{CHWs}$ could expand the capacity of local medical teams to move the vaccines more quickly throughout each country, thus multiplying the workforce and dividing the time needed to distribute the shots. The WHO training course covers the spectrum of required tasks, including cold-chain requirements, infection control measures, adverse events after the jab, the paperwork required for vaccinations, and communicating with communities about the need for and the safety of COVID-19 vaccines. It is a valuable course, offered in ten languages (with more promised); it takes three hours to complete. 
Preparing $\mathrm{CHWs}$ to expand the vaccination workforce is an excellent idea; however, the numbers of $\mathrm{CHWs,}$ like the numbers of physicians and nurses, fall short of the labor force needed to get the vaccines into large populations quickly. By even the loosest definition of $\mathrm{CHW}$, there are just too few of these paraprofessionals in the places they would be most needed to flesh out vaccination teams on the ground. Consider that in Central America, the Caribbean, and portions of South America, there are only $10.6 \mathrm{CHW}$ ser 10,000 people. In SubSaharan Africa, there are $4.3 \mathrm{CHWs}$ per 10,000. ${ }^{19}$ Teams of physicians, nurses, and CHWs will not be adequate for rapid deployment and the administration of vaccinations throughout urban and rural areas of low- and middleincome countries.

The question then becomes: Even when vaccines become available, will these underserved regions find the vaccinators to administer them?

\section{AN INNOVATIVE SPIN-OFF OF THE WHO VACCINATION TRAINING PROGRAM}

"We urge African nations to ramp up readiness and finalize their national vaccine deployment plans. . cold chain systems and distribution plans need to be in place to ensure vaccines are safely expedited from ports of entry to delivery. We can't afford to waste a single dose." 20

- Dr. Matshidiso Moeti, WHO Regional Director for Africa

Dr. Moeti's advice for vaccine administration in Africa holds as well for all underserved regions with limited medical staffs and related human resources needed to distribute vaccines safely without wasting a single dose. Given the rapid spread of COVID-19 and the dangers of variants arising in unvaccinated populations, it is also important to administer vaccines quickly. Each vaccinated person deprives the virus of an opportunity to mutate into a threatening variant, and so countries must plan for accelerated vaccination schedules. This means staffing up to reach even distant communities with vaccinators who can observe cold chains and other distribution requirements, as well as getting the jabs into the arms of many thousands of people as rapidly as possible.

As we have seen, there are just not enough medical professionals in many underserved regions to do the job, a shortfall recognized by $\mathrm{WHO}$ as it prepared its $\mathrm{CHW}$ vaccinator program. Thus, the plan involves current medical professionals, CHWs, and even retired doctors and nurses recalled into the service of this massive vaccination effort. Yet, the numbers remain inadequate in many low- and middle-income countries. As we have seen, even the CHW force, while helpful for sure, is not sufficient to create an adequate army for the vaccination rollout.

\section{RECRUITING CITIZEN VACCINATORS}

\section{A Community Health Worker Model}

Two years ago, WiRED developed a training program for low-resource regions that prepares people with at least a high school diploma to serve as CHWs. ${ }^{21}$ The five-week course, taught in person by medical professionals, covers a wide range of health, medical and communication topics. The course, developed by a team of medical professionals, has been peer-reviewed and tested in four countries. The curriculum, which takes approximately five weeks to complete, is WHO compliant.

This CHW program successfully demonstrated that a well-designed, evidence-based curriculum taught by professional medical instructors could effectively train local people to serve as CHWs. In fact, one of the first classes to earn CHW certificates early in 2020 immediately stepped into their roles by providing health services as COVID-19 overwhelmed conventional medical resources. WiRED tracked these CHWs for a full year and recorded their successful activities that provided clinical services, prevention training, referrals, and other assistance to tens of thousands of members of their communities at a time when doctors and nurses were overworked and far too few in number to address the health and medical needs of COVID-19 impacted communities.

\section{Vaccinator Training Program}

Our experience with the $\mathrm{CHW}$ training program points the way to training teams of community vaccinators. As we have noted, the health administrators in low-resource countries will face vaccine shortfalls and a vaccination workforce shortage when vaccines arrive. Too few vaccinators will hamper the swift and effective delivery of the vaccine to large populations.

WiRED assembled a curriculum development team in late 2020 to create a comprehensive COVID-19 vaccination course designed for people who had at least a high school diploma. Success with our CHW training program convinced 
us that a course focusing on COVID-19 vaccinations could provide the workforce needed to augment the efforts of medical professionals. The certificate earners would work in teams, side-by-side with the doctors and nurses who directed shot clinics. To prepare them for this work, vaccination students would become familiar with each component of the $\mathrm{WHO}$ vaccinator training curriculum along with key health topics in our CHW training program.

While the WHO curriculum serves as the backbone of WiRED's Vaccinator Training Program, there are significant differences between the two. The driving difference is that the WHO's program trains CHWs, while WiRED's program reaches beyond $\mathrm{CHWs}$ and trains local people who have no medical backgrounds. Local vaccinator training can add thousands of qualified people to supplement the vaccination workforce. Table 1 compares the two programs:

\section{WIRED'S VACCINATOR TRAINING PROGRAM}

Let's now consider WiRED's Vaccinator Training Program, examine the curriculum, teaching methods, and how the certificate earners would assist in vaccinating populations against COVID-19.

\section{The Curriculum}

The WiRED Vaccinator Training Program (VTP) is available online and for downloading. Using a special app provided by WiRED, instructors and students download the complete course to their laptops, tablets, or smartphones. The course has two sections. The first tracks the WHO curriculum very closely (Modules 1-3 and 5-7). WiRED enhances the WHO material with explanations, illustrations, examples, and other features that enrich the content for people not medically trained. The second section, designed initially for WiRED's CHW program, teaches students essential human anatomy and physiology, infectious disease, patient assessment, and communication. We will look at the communication training more closely later in the paper.

For the WHO curriculum material, WiRED adds embedded questions and answers that follow best learning theory practices. These interactive Q\&As reinforce key concepts presented throughout the module, which helps students understand the material and retain it. To gauge the students' grasp of the material and to reinforce the key concepts one more time, course developers have added a comprehensive final exam to each module.

WiRED developed Module \#4 specifically for people new to vaccine administration. "Vaccination Process and Procedures" presents detailed steps to administer an intramuscular injection; prepare the equipment; prepare single- and multi-dose vials; position the patient correctly, and dispose of waste appropriately. This detailed module offers checklists, descriptions, and videos that show each procedure in detail.

Lessons presented in Module \#4 then move from the classroom to the clinic, where the instructor provides students with five hours of injection preparation and administration practice. Students proceed through the sequence many times to be certain they can implement the procedures without error. Most low-resource locations are unlikely to have simulation manikins, so students practice giving shots by injecting oranges, a method used for many years in nursing and medical schools.

The following topics comprise the core training program; Table 3 provides a summary of each topic. Note again that topics presented in Modules 1-3 and 5-7 align with the WHO Vaccinator curriculum, while WiRED created_Module \#4.

Module 1: Introduction to vaccination training Module 2: Storage, handling, delivery, and waste management of vaccines

Module 3: Organizing vaccination sessions Module 4: Vaccinating process and procedures Module 5: AEFI monitoring for vaccination Module 6: Recording and monitoring vaccination

Module 7: Communication with the community about vaccination

As we noted, our VTP students study five additional health modules from WiRED's CHW curriculum:

- Anatomy Fundamentals, two parts

- Understanding Communicable Diseases, two parts

- Coronavirus Review

- Understanding Vital Signs and Basic Assessment of the Systems

\section{- Communication Skills}

The first four topics concern medical issues that relate to the vaccinators' clinical responsibilities. Communication Skills, on the other hand, may seem peripheral to the vaccinators' job description. A critical part of the community vaccination process is to teach residents, many of whom will be skeptical of vaccinations, about the COVID-19 virus, how the disease is spread and why vaccinations are critical 
in stemming the spread of infection. Reluctant community members can defeat vaccination efforts and undermine goals to vaccinate large swaths of the public needed to reach herd immunity. Thus, communication becomes as important as any other element in this curriculum. While WHO's, Module \#7 covers communication, WiRED saw an opportunity to expand coverage of the topic. Many local vaccinators will likely be required to approach communities as advocates of the vaccination process.

\section{An Additional Training Element}

As vaccines arrive in low- and middle-income countries, communities will want trained vaccinators waiting, springloaded to distribute the vaccine and begin vaccinations. Training people in advance is wise, but it comes with the risk that if supplies are delayed for long, vaccinators in waiting will forget critical details of the work. To minimize the risks of long delays, WiRED requires all certificate earners to enroll in a continuing medical education (CME) program, available online through a WiRED app. Each month, vaccinators are obliged to review refresher material to sustain their familiarity with details. The app links vaccinators to the training modules, which then allows them to keep track of their activity. This vaccinator CME program has been appended to WiRED's larger CME program for $\mathrm{CHWs}$, who are required to earn 50 credits each year to maintain their credentials.

\section{AN INITIAL TEST OF THE VTP}

WiRED completed curriculum development in midFebruary 2021 and enrolled a group of 26 people to take the 45-hour class, instructed by a physician and a nurse. The class was held in Kisumu, a city of over a half-million people on the shore of Lake Victoria in northwestern Kenya, not far from the Ugandan border. The training was conducted in a large slum, where many students live and will eventually work as vaccinators. The classroom training and the clinical practice session went as planned, and 24 students scored above $85 \%$ on the exams included at the end of each module. The certification exam, which will be administered online in late spring, will not be completed in time for this article. Students also completed a course evaluation form where they reported great enthusiasm for the course. They viewed the course as appropriate for the tasks ahead, that it was interesting and thorough, and had prepared them for their important work. The overwhelming majority of students also felt strongly that their family and community would be proud of them as vaccinators and that their new skills would benefit their communities.

\section{CONCLUSION}

The global population had lived under a cloud of the COVID-19 pandemic for the better part of a year when news began to surface about successful early trials of a vaccine. Messenger RNA vaccines, two of the earliest to clear Phase 3 trials, were based on a new and promising technology, known in laboratories for more than two decades but never employed on such a scale against a virus that threatened life on the entire planet.

In December 2020, the mRNA vaccines and vaccines based on other technologies (e.g., non-replicating viral vaccines and inactivated viruses) began to roll out in high-income countries. Word of their success filled headlines, and people lined up to receive their jabs with the expectation that life would return to near-normal for them and their families. Return to normalcy is taking longer than most expected, and that's due in part to the supply of vaccines and in part to the acceptance of vaccines in some demographic groups. Normalcy, in this case, depends as much on the immunity of the larger society as it does on the immunity of the individual. At the time of this writing, significant portions of the population in high-income regions enjoy some measure of protection against the virus.

This isn't so for low- and middle-income countries. Large, transnational organizations deliver vaccines to low-resource 
regions, but the work is slow, and vaccine supplies are limited. In Kenya, for instance, COVAX delivered one million doses of the AstraZeneca-Oxford vaccine, ${ }^{22}$ and it was quickly distributed among healthcare workers and others whose jobs are considered essential. However, the 52 million other Kenyans will need to wait, perhaps until $2023,{ }^{23}$ because there simply is not enough vaccine to go around in their country or other low-resource regions.

We know the vaccines will arrive someday, perhaps after vaccines saturate high-income regions, and leaders in these countries recognize the validity of the refrain: no one is safe until everyone is safe. As long as large swaths of the global population remain unvaccinated, the virus enjoys a reservoir of people in whom they can thrive and generate variants that continue to threaten all human life. Eventually, as the existing versions and newly approved vaccines ramp up production, ample doses will arrive throughout the world.

That's where the next problem will arise: With billions of people standing in line for vaccinations, who will vaccinate them? We know there are not enough doctors or nurses, or $\mathrm{CHWs}$. And so, a vaccination logjam will form in low- and middle-income countries, delaying the intervention that can limit the rekindling of this pandemic.

Anticipating a shortfall in the number of vaccinators, WiRED developed a curriculum, based on the WHO vaccinator program, that would make available a largely new crew of qualified vaccinators to join physicians and nurses that deploy to the cities and countryside to administer the shots. These citizen vaccinators are trained to transport vaccines, teach communities about the virus and vaccinations, set up shot clinics, administer the jabs and monitor patients for AEFI. These vaccinators come from the communities they serve, so they know the people and the customs; accordingly, they are trusted over vaccinating teams from the outside. And the vaccinators know the science and the technical procedures necessary for administering vaccinations. These vaccinators are force multipliers, expanding the understaffed medical corps' capacity to lead the vaccination outreach efforts.

In one 45-hour week, the training program can provide the necessary skills to these citizen vaccinators, who will stand ready to deploy as soon as vaccines arrive. WiRED has provided community health training for more than two decades, and it has trained $\mathrm{CHWs}$, people drawn from the communities they serve. This experience has enabled the organization to step in at a time when a large vaccinator corps will be needed to help vaccinate entire populations quickly. These citizen vaccinators will help save not only people in lowresource regions but people throughout the world.

\section{TABLE 3.}

TRAINING MODULES

Module 1: Introduction to vaccination training. This module offers an introduction to the course, whose objective is to provide key information on vaccination to community health workers.

Module 2: Storage, handling, delivery, and waste management of vaccines. This module consists of three sections, which provide knowledge about the programmatic implications of vaccines; managing storage, transport, and delivery of vaccines; and cold-chain management at the vaccination post and waste management at the facility level.

Module 3: Organizing vaccination sessions. This module explains how to: outline infection prevention and control measures during vaccination sessions to protect health workers, vaccine recipients, and the community; prepare for vaccination sessions according to infection prevention and control protocols (at the health facility, for outreach, and a campaign); and outline the process of safe vaccine administration and waste disposal.

Module 4: Vaccinating process and procedures. This module explains how to: administer an intramuscular injection, prepare the equipment, prepare single- and multi-dose vials, position the patient correctly, and dispose of waste appropriately.

Module 5: AEFI monitoring for vaccination. This module describes how to: detect an adverse event following immunization (AEFI); identify and respond to AEFI, and report AEFI following vaccination.

Module 6: Recording and monitoring vaccination. This module describes the importance of proper recording and reporting, recognizing recording and registration forms, correctly recording vaccination data and register vaccinations in the reporting forms, and tracking defaulters (if applicable).

Module 7: Communication with the community about vaccination. This module describes community health workers' roles and responsibilities for vaccination; demonstrates effective and individualized communications about vaccination; explains communication strategies for three potential scenarios with community members; and outlines the role of a vaccinator crisis communication for adverse events following immunization. 


\section{TABLE 4.}

\section{SUPPLEMENTAL TRAINING}

1.2.1 Anatomy Fundamentals, part 1. Part 1 provides an understanding of anatomy and physiology, which together comprise the structure and functions of the human body, and describes the major body systems and their components.

1.2.2 Anatomy Fundamentals, part 2. Part 2 discusses the body's major systems: the nervous system, the respiratory system, the digestive system, the urinary system, the endocrine system, the lymphatic system, and the male and female reproductive systems.

1.3 Understanding Communicable Diseases, two parts. This two-part module presents an overview of infection control, preparedness, and modes of transmission and discusses the role of $\mathrm{CHWs}$ in addressing infectious diseases.

1.6 Coronavirus. The module offers a general introduction to the new coronavirus (COVID-19) and describes the infection and its close relationship to other coronaviruses, how it is spread, its incubation period and symptoms, treatment, and most important - its prevention.

2.1 Understanding Vital Signs and Basic Assessment of the Systems. This module examines basic procedures for taking vital signs, including temperature, pulse rate, blood pressure, and respiration rate. It offers "how-to" information and explains how each measure relates to health assessment.

3.1 Communication Skills. This module covers interpersonal and group communication, persuasion, relationship building, message development, and other skills necessary to CHWs as communicators.

\section{AUTHOR DISCLOSURES:}

No relevant financial affiliations or conflicts of interest.

\section{REFERENCES:}

1. There are four types of vaccines: Here's how they work. Gavi website. https://www.gavi.org/vaccineswork

2. Katella K. Comparing the vaccines: How are they different? Yale Medicine website (2021 Apr 5). https://www. yalemedicine.org/news/covid-19-vaccine-comparison

3. CDC Recommends Use of Johnson \&amp; Johnson COVID-19 Vaccine Resume https://www.cdc.gov/ coronavirus/2019-ncov/vaccines/safety/JJUpdate.html

4. Joint CDC and FDA Statement on Johnson \& Johnson COVID-19 Vaccine. https://www.cdc.gov/ media/releases/2021/s0413-JJ-vaccine.html

5. FDC and CDC Lift Recommended Pause on Johnson \& Johnson (Janssen) COVID-19 Vaccine. https://www. fda.gov/news-events/press-announcements/fdaand-cdc-lift-recommended-pause-johnson-johnsonjanssen-covid-19-vaccine-use-following-thorough

6. Coronavirus (COVID-19) vaccinations. Our World in Data website. https://ourworldindata.org/covid-vaccinations
7. https://www.state.gov/secretary-antony-j-blinkenremarks-to-the-press-on-the-covid-response/

8. There are four types of vaccines: Here's how they work. Gavi website. https://www.gavi.org/vaccineswork

9. COVID vaccine rollout: Which country in Europe is leading the race? Euronews website. https://www. euronews.com/2021/03/26/covid-19-vaccinationsin-europe-which-countries-are-leading-the-way

10. There are four types of vaccines: Here's how they work. Gavi website. https://www.gavi.org/vaccineswork

11. vaccine tracker. COVID19 Vaccine Tracker website. https://covid19.trackvaccines.org/vaccines

12. COVID vaccine rollout: Which country in Europe is leading the race? Euronews website. https://www. euronews.com/2021/03/26/covid-19-vaccinationsin-europe-which-countries-are-leading-the-way

13. COVAX: Working for global equitable access to vaccines. World Health Organization website. https:// www.who.int/initiatives/act-accelerator/covax

14. Nelson C. The case for equitable vaccine distribution. International Finance Corporation website. https:// www.ifc.org/wps/wcm/connect/news_ext_content/ ifc_external_corporate_site/news+and+events/ news/insights/equitable-vaccine-distribution

15. Lambert J. Global inequity in vaccination is more than a moral problem. Science News. 2021 Mar 27;199(6). https://www.sciencenews.org/article/covid-19-globalinequity-vaccines-deaths-economy-pandemic

16. Carter C, Anh NTL, Notter J. disease: Perspectives in lowand middle-income countries. Clinics in Integrated Care. 2020 Jul 17;1:100005. doi:10.1016/j.intcar.2020.100005. https://www.ncbi.nlm.nih.gov/pmc/articles/PMC7261656

17. Nurses and midwives (per 1,000 people). The World Bank website. https://data.worldbank. org/indicator/SH.MED.NUMW.P3.

18. Vaccination training for health workers. World Health Organization website. https://openwho.org/ courses/covid-19-vaccination-healthworkers-en

19. Global Health Observatory data repository. World Health Organization website. ealth Observatory data repository.https://apps.who.int/gho/data/node. main.HWFGRP_0180?lang=en Population, total. The World Bank website. https://data.worldbank.org/ indicator/SP.POP.TOTL?end $=2019 \&$ start $=2004$

20. Dispatch of millions of vaccines to Africa expected to start in February: WHO. UN News website (2021 Feb 5). https://news.un.org/en/story/2021/02/1083882

21. Selnow G, Othman M, Crano W. Introduction and follow up of ongoing WiRED International program. Medical Journal of Southern California Clinicians. 2020 May/Jun; 13(1):30-37. http://socalclinicians.org/periodical/wired.pdf

22. Over one million vaccine doses arrive in Nairobi, via COVAX facility. UNICEF website (2021 Mar 2). https://www.unicef.org/ kenya/press-releases/over-1-million-covid-19-vaccine-dosesarrive-nairobi-via-covax-facility

23. The ins and outs of Kenya's vaccine rollout plan. Gavi website. https://www.gavi.org/vaccineswork/ins-and-outs-kenyas-covid19-vaccine-rollout-plan 